\title{
Regional Dissemination of a Carbapenemase- encoding Plasmid: Plasmidome analysis Reveals Diverse Adaptations of Carbapenemase-Producing Enterobacteriaceae
}

\section{Ryuichiro Abe}

Osaka University

Yukihiro Akeda ( $\square$ akeda@biken.osaka-u.ac.jp )

Osaka University https://orcid.org/0000-0002-5325-4173

\section{Yo Sugawara}

Osaka University

Dan Takeuchi

Osaka University

\section{Yuki Matsumoto}

Osaka University

\section{Daisuke Motooka}

Osaka University

Norihisa Yamamoto

Osaka University

\section{Ryuji Kawahara}

Osaka Institute for Public Health

\section{Kazunori Tomono}

Osaka University

\section{Yuji Fujino}

Osaka University

Shigeyuki Hamada

Osaka University

\section{Research}

Keywords: heteroresistance, carbapenemase, carbapenem resistance, plasmid dynamics, plasmidome, plasmid analysis, IMP-6, horizontal dissemination, chromosomal integration, Enterobacteriaceae

Posted Date: June 15th, 2020 
DOI: https://doi.org/10.21203/rs.3.rs-34719/v1

License: (c) (i) This work is licensed under a Creative Commons Attribution 4.0 International License. Read Full License 


\section{Abstract}

Background. The global dissemination of carbapenem-resistant Enterobacteriaceae (CRE) threatens human health by limiting the range of usable antibiotics even against common bacterial infections. The spread of CRE is primarily due to the transmission of carbapenemase genes located on plasmids. However, few studies have comprehensively identified regionally spreading carbapenemase-encoding plasmids because of the difficulty to determine the complete sequence of a plasmid encoding carbapenemases. In a CRE surveillance study of 1,507 patients from 43 hospitals in northern Osaka, Japan, we previously found that $12 \%$ of the patients carried CRE and $95 \%$ of CRE isolates were IMP- 6 producers. This result suggested a vast horizontal spread of a clonal plasmid carrying $b / a_{\mid \mathrm{MP}-6}$ among Enterobacteriaceae in this region. In the current study, we aimed to describe the dynamics of this regional horizontal plasmid transmission.

Results. We systematically analysed the plasmids of $230 \mathrm{CRE}$ isolates carrying b/a $a_{\mathrm{MP}}$ obtained in our previous surveillance study by using whole genome sequencing and Southern blotting. We detected a major population (187 out of 230 b/a $a_{\mid \mathrm{MP}}$-positive CRE isolates, $85.6 \%$ ) that carried $b / a_{\mathrm{IMP}-6}$ on the IncN plasmid pKPI-6, along with diverse minor subpopulations. Among the subpopulations, we identified a novel cluster carrying an IncF plasmid that leads to heteroresistance due to amplification of $b / a_{1 \mathrm{MP}-6}$, resulting in covert transmission of $b / a_{\mid \mathrm{MP}-6}$ or occasional chromosomal integration of $b / a_{\mid \mathrm{MP}-6}$. In addition, we detected one isolate that harboured $b / a_{\mid \mathrm{MP}-1}$, which is identical to $b / a_{\mid \mathrm{MP}-6}$ except for a single point mutation, on pKPI-6 and thus had acquired a broader range of antimicrobial resistance.

Conclusions. Carbapenemase-encoding plasmid tracking revealed the clonal dissemination of pKPI-6 among chromosomally distinct isolates. Focusing on the mode of carbapenemase gene carriage is helpful for monitoring of horizontal spread of CRE isolates that is difficult to trace only by the comparisons of the whole genomes. A seemingly clonal horizontal dissemination of the predominant plasmid had embraced heterogenous subpopulations that contribute to diverse adaptations including covert transmission, stable chromosomal integration of $b / a_{\mathrm{IMP}-6}$, or broadened antimicrobial resistance patterns, ultimately leading to treatment failure.

\section{Introduction}

The rapid global dissemination of multidrug-resistant Enterobacteriaceae threatens healthcare systems worldwide [1]. Carbapenem-resistant Enterobacteriaceae (CRE) are of major concern because alternative treatment options are limited [2]. Carbapenem resistance is primarily conferred by carbapenemases, which are enzymes that hydrolyse carbapenem [3]. KPC, NDM, and OXA-48 are the most commonly detected carbapenemases [3]. Carbapenemase genes are generally plasmid-encoded and are frequently transmitted across species [4]. Genetic tracking of plasmids encoding carbapenemase genes has allowed monitoring the spread of $\mathrm{CRE}$ isolates. For example, structural similarities among plasmids from isolates obtained in a single hospital outbreak allowed elucidating links between patients carrying the isolates [57], and plasmid data accumulated globally revealed the worldwide spread of an epidemic plasmid 
carrying b/a $a_{\mathrm{KPC}}$.[8]. However, most regional surveillance studies compared the whole genomes of CRE isolates without analysing the clonality of the spreading carbapenemase-encoding plasmids, and few studies have comprehensively analysed regionally spreading carbapenemase-encoding plasmids in order to reveal the modes of horizontal plasmid transmission in a certain region [9].

We previously conducted a surveillance study of CRE in 1,507 patients from 43 hospitals in northern Osaka (population: 1,170,000, area: $307 \mathrm{~km}^{2}$ ), Japan [10], and we reported that $12 \%$ of the patients carried CRE and $95 \%$ of CRE isolates harboured $b / a_{\mathrm{IMP}-6}$, the predominant carbapenemase in Japan. The predominance of this particular carbapenemase gene might have resulted from vigorous horizontal spreading of a specific plasmid carrying bla ${ }_{\mathrm{IMP}-6}$, pKPI-6 [11], in this region. The aim of the current study was to profile the mode of carriage of carbapenemase genes, primarily $b / a_{\mathrm{IMP}}$, to unveil their diversity within a defined geographical region.

\section{Results}

\section{Dissemination of pKPI-6}

All bla $a_{\mathrm{IMP}}$-positive CRE isolates of Escherichia coli $(\mathrm{n}=135)$ and Klebsiella pneumoniae $(\mathrm{n}=95)$ were classified into seven groups based on the results of S1-PFGE followed by Southern blot hybridization with probes for the bla $a_{\mathrm{IMP}}$ and repA genes (Figure 1). Ninety-nine of the $135 \mathrm{E}$. coli isolates (73\%) and 88 of the 95 K. pneumonia isolates (93\%) carried plasmids classified as Group pKPI-6 based on plasmid size and replicon type (Supplementary Figure S1). pKPI-6 was the predominant plasmid responsible for the transmission of $b / a_{\mathrm{IMP}-6}$ (187 out of 230 bla $\mathrm{IMP}_{\mathrm{IMP}}$-positive CRE isolates, $85.6 \%$ ). Next, we compared the similarity between pKPI- 6 and 39 representative plasmids categorized as Group pKPI-6 based on WGS data. The overall sequence identity was $99 \pm 0.28 \%$, and the sequence coverage was $98 \pm 4.0 \%$ (mean \pm standard deviation) (Supplementary Figure S1). This analysis confirmed that pKPI-6 is the predominant plasmid carried by CRE isolates in the study area.

\section{Genomic Analysis of Derivatives of the Predominant Plasmid, pKPI-6}

During the characterization of the bla $a_{\mathrm{MP}-6}$ plasmids mentioned above, nine $E$. coli isolates and three $K$. pneumoniae isolates possessed bla $a_{\mathrm{IMP}-6}$ plasmids categorized as Group IncN (Figure 1). Group IncN $b / a_{\mathrm{IMP}-6}$ plasmids were characterized by replicon type $\mathrm{IncN}$ and ranged from 35 to $264 \mathrm{kbp}$ in size, which was different from the pKPI-6 plasmid of $50 \mathrm{kbp}$ (Supplementary Figure S1). The complete sequences of these plasmids indicated that they had preserved the nearly complete locus of pKPI- 6 and typically were multi-replicon plasmids that had integrated IncF-type plasmids framed by insertion sequences (Supplementary Figure S2 and Table S1). Additionally, two isolates (E208 and E328) of K. pneumoniae harboured plasmids categorized as Group Non-IncN KP (Figure 1B). These plasmids comprised a cassette carrying $b / a_{\mid \mathrm{MP}-6}$ without IncN-type repA of the pKPI-6 plasmid integrated into another plasmid (Supplementary Figure S3). Interestingly, E. coli isolate E119 and K. pneumoniae isolate E206 
coharboured two distinct $b / a_{\mathrm{IMP}-6}$-encoding plasmids of different sizes and were categorized as Group double bla $\left.\right|_{\mathrm{IMP}-6}$ (Figure 1 and Supplementary Figure S4). Barring occasional isolations of strains coharbouring different carbapenemase genes [12,13], few studies have shown the coexistence of two identical carbapenemase genes on different plasmids within an isolate [14]. WGS revealed that isolate E119 carried pKPI-6 and an IncF-type plasmid (pEC743_1) that had a b/a $a_{\mid \mathrm{MP}-6}$ cassette from pKPI-6 integrated (Supplementary Figure S5).

\section{Characterization of IncF Plasmids Encoding bla ${ }_{\mathrm{IMP}-6}$}

In addition to the K. pneumoniae isolates carrying Group Non-IncN KP plasmids, E. coli isolates carrying plasmids without IncN replicon were found in a single hospital (hospital D; Figure 1A). WGS of these isolates revealed that they harboured nearly identical b/a $a_{\text {IMP- } 6}$-encoding plasmids with an IncFIA-type replicon (categorized as Group IncF) (Supplementary Figure S6A and Table S1). These plasmids were generated by integration of a cassette carrying $b / a_{\mathrm{IMP}-6}$ on pKPI-6 into another IncF plasmid at IS 26 . This IncF plasmid (pEC302/04; Supplementary Figure S6B) has been reported to transmit antimicrobial resistance since 1965 [15].

The minimum inhibitory concentrations (MICs) of meropenem for the $E$. coli isolates carrying Group IncF plasmids were low when compared with those of $E$. coli isolates harbouring other $b / a_{1 \mathrm{MP}-6}$-encoding plasmids, such as pKPI-6 (Supplementary Figure S7). Mutations or deletions in the porin (OmpF) gene in E. coli have been reported to enhance resistance to $\beta$-lactams [16]. However, all E. coli isolates carrying Group IncF plasmids had a premature termination codon within $\mathrm{ompF}$, whereas the other isolates carried wild-type ompF (Supplementary TableS2 and S3). MICs of meropenem were low for these Group IncF plasmid-carrying isolates, despite them being OmpF-deficient. To investigate carbapenem resistance in the same genetic background, plasmids from representative isolates in each $b / a_{\mid \mathrm{MP}-6}$ carriage group were transformed into the E. coliTOP10 strain and MICs for the transformants were determined. Transformant T305 carrying pE305_IMP6 $6_{\text {single }}$ of Group IncF from E. coli isolate E305 was more susceptible to meropenem than transformants carrying $b / a_{\mathrm{IMP}-6}$-harbouring plasmids of groups (Supplementary Table S4). bla $a_{\mathrm{MP}-6}$ transcription in the pE305_IMP6 $6_{\text {single }}$ transformant was significantly lower than that in the pKPI-6 transformant (Supplementary Figure S8A), although the plasmid copy numbers in the bacterial cells were comparable (Supplementary Figure S8B). These results indicated that the lower MICs of meropenem in $E$. coli isolates carrying Group IncF plasmids were due to reduced transcription of $b / a_{\mathrm{IMP}-6}$.

\section{Heteroresistance to Carbapenems: Enhanced Resistance Through Gene Amplification}

E. coli isolates E305 and E318 were found to carry Group IncF plasmids, and WGS revealed that their chromosomes were nearly identical (query: E318, identity $100 \%$, coverage $100 \%$; query: E305, identity $100 \%$, coverage $98 \%$ in BLASTN). Isolate E318 harboured genes encoding extended-spectrum $\beta$ lactamases (ESBLs), such as bla $a_{\mathrm{CTX}-\mathrm{M}-14}$ and $b / a_{\mathrm{TEM}-1 \mathrm{~B}}$, on a plasmid other than pE318_IMP6, whereas isolate E305 did not have these genes (Supplementary TableS5). IMP-6 confers resistance to 
cephalosporins and meropenem, but it hydrolyses penicillins very poorly [17]. Therefore, isolate E318 exhibited broader antimicrobial resistance than isolate E305. In contrast, the MIC of meropenem for E305 was higher than that for E318.

WGS of E305 and E318 revealed the complete sequence of pE318_IMP6; however, it failed to determine the complete sequence of pE305_IMP6. Therefore, to analyse the structure of pE305_IMP6, we used a combination of WGS, Southern blotting, and qPCR analysis. The length and depth of each contig of pE305_IMP6 deduced from WGS are shown in the de-novo assembly graphs generated using the Bandage software [18] in Figure 2A. The total length of pE305_IMP6 deduced from WGS data was approximately $149 \mathrm{kbp}$. However, according to Southern blotting results, pE318_IMP6 and pE305_IMP6 were $\sim 145 \mathrm{kbp}$ and $\sim 200 \mathrm{kbp}$ in size, respectively (Figure 2B). Based on the depth of each contig, the copy number of each contig was predicted as follows: Contig3, 1 copy; Contig2 and Contig5, 6 copies; Contig1 and Contig6, 3 copies; Contig4, 5 copies (Figure 2A). Therefore, pE305_IMP6 was predicted to have a $\sim 19-k b p$ repeat region consisting of triplication of Contig1 and Contig6, sextuplication of Contig2 and Contig5, and quintuplication of Contig4 (Figure 2C). Except for the repeat region, pE305_IMP6 and pE318_IMP6 exhibited high sequence similarity (identity; 99.27\%, coverage; 100\%) (Figure 2D). The b/a $a_{\text {IMP- } 6}$ gene was located on Contig6 and was predicted to be triplicated. QPCR analysis corroborated that pE305_IMP6 carried three copies of b/a IMP-6, whereas pE318_IMP6 harboured a single copy (appendix p10). bla $a_{\mathrm{IMP}-6}$ transcription was significantly higher in isolate E305 than in isolate E318 (Figure 2E), even though the $b / a_{\mid \mathrm{MP}-6}-$-carrier plasmid copy numbers in the cells of these isolates were not significantly different (Supplementary Figure S9B). Triplication of $b / a_{\mid \mathrm{MP}-6}$ in tandem resulted in a higher transcription level in E305, resulting in a higher level of resistance to meropenem.

Subculture of the clonal isolate E305 in broth medium revealed a mixture of subpopulations of bacteria carrying a plasmid with multiple b/a $a_{\mathrm{MP}-6}$ copies (which represented the majority) and bacteria carrying a plasmid with a single $b / a_{\mathrm{IMP}-6}$ copy. In Southern blotting for $b / a_{\mathrm{IMP}-6}$, a faint band at $\sim 145 \mathrm{kbp}$ was observed in addition to the major band at $200 \mathrm{kbp}$ (Figure 2B). It was also found that T305 (transformant of pE305_IMP6 single extracted from E305) carried a 145-kbp plasmid without bla IMP-6 $_{1}$ amplification due to recA deficiency in the recipient E. coli TOP10 strain (Supplementary Figure S10) [19]. qPCR analysis confirmed that T305 carried one bla IMP-6 $_{1}$ copy on its plasmid (Supplementary Figure S9C).

These results indicated the existence of a subpopulation carrying a plasmid with one b/a $a_{\text {MP- } 6}$ copy within E. coli isolate $\mathrm{E} 305$, whereas the majority of the population carried a plasmid harbouring three copies of bla $a_{\mid \mathrm{MP}-6}$.

\section{Comparison of CRE Isolates Carrying pKPI- 6 with Those Carrying Other Groups of Plasmids Harbouring bla $a_{\mathrm{MP}-6}$}

bla $a_{\mathrm{CTX}-\mathrm{M}-2}$, which is an ESBL gene located distant from $b / a_{\mathrm{IMP}-6}$ on $\mathrm{PKPI}-6$, compensated for the narrow range of hydrolysis of $\beta$-lactams by IMP-6 $[11,17]$. However, toweverhhhhese two $\beta$-lactamase genes were not always transferred together from pKPI- 6 to another plasmid. Plasmids categorized as Group 
Non-IncN KP and Group IncF did not carry ESBL genes (Supplementary Table S6) and rarely conferred resistance to penicillins, in contrast to pKPI-6, which confers broad resistance to $\beta$-lactams (Figure 1). We next measured the conjugation efficiency of representative plasmids in each group (Supplementary Table S7). pKPI-6 plasmids and Group IncN plasmids, which had the entire pKPI-6 plasmid incorporated, showed a higher conjugation efficiency than Group Non-IncN KP/IncF plasmids. These characteristics may have facilitated the vast horizontal dissemination of pKPI-6 in the study area.

Compared with the chromosomal diversity among E. coli isolates bearing pKPI-6, K. pneumoniae isolates carrying pKPI-6 exhibited higher clonality as indicated by Xbal-PFGE analysis (Figure 1). This may be explained by the presence of the $k i k A$ gene on pKPI-6, the product of which reportedly promotes cell death of K.pneumoniae following conjugation [20]. The conjugation efficiency of pKPI-6 into K. pneumoniae ATCC13883 was considerably lower than that into E. coli TUM3456 $\left(3.3 \times 10^{-4}\right.$ and $3.7 \times 10^{-1}$, respectively). Maybe only "kikA-resistant" K. pneumoniae are able to acquire pKPI-6, leading to clonal similarity among the $K$. pneumoniae isolates bearing $\mathrm{pKPI}-6$.

\section{Chromosomal Integration of $b / a_{\mathrm{IMP}-6}$}

Unlike most CRE isolates, which carried the predominant pKPI- 6 or other $b / a_{\mid \mathrm{MP}-6}$-encoding plasmids, three out of $135 \mathrm{E}$. coli isolates (E138, E300, and E302) harboured b/a ${ }_{1 \mathrm{MP}-6}$ on their chromosomes as indicated

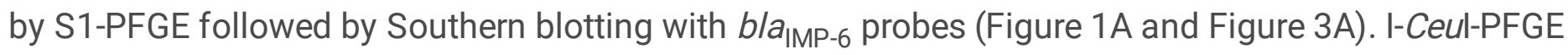
followed by Southern blotting with probes for the bla $a_{\mid \mathrm{MP}-6}$ and $16 \mathrm{~S}$ rRNA genes confirmed chromosomally located $b / a_{\text {IMP- } 6}$ (Figure 3B). WGS revealed that the chromosome of isolate E138 had a cassette harbouring b/a $a_{\text {IMP- } 6}$ integrated, framed by a set of IS 15 (Figure 3C). The chromosomes of E300 and E302 had IncFIA plasmids carrying b/a $a_{\text {MP-6 } 6}$ integrated (Figure 3D,E). While these plasmids were essentially identical to pE301_IMP6 (E. coli, Group IncF), these isolates were phylogenetically distinct on the XbalPFGE phylogenetic tree (Figure 1).

\section{Emergence of pKPI-6-like Plasmid Harbouring bla $a_{\mathrm{MP}-1}$}

One K. pneumoniae isolate, E105, harboured $b / a_{\mid \mathrm{MP}-1}$, which is a single-nucleotide variant of $b / a_{\mid \mathrm{MP}-6}$, within a clonal cluster of pKPI-6 carriers (Figure 1B). Due to this mutation, E105 was resistant to imipenem, whereas most isolates carrying $b / a_{\mid \mathrm{MP}-6}$ were susceptible to this antibiotic. WGS revealed that plasmids pKPI-6, pE013_IMP6 (plasmid group pKPI-6), and pE105_IMP1 were 99.8\% identical, with a coverage of 100\% (query: pE013_IMP6) (Figure 4). The only difference was the presence of a 714-bp region bracketed by a set of homologous regions in pE013_IMP6.

\section{Discussion}

IMP-producing Enterobacteriaceae have been reported sporadically on a global basis [2]. IMP-4-producing Enterobacteriaceae are endemic to Australia [21], and IMP-1-, 4-, and 8-producers have been occasionally detected in China [22]. Our study revealed the exclusive dissemination of IMP-6 producers (95\% of CRE 
isolates) in northern Osaka, Japan, consistent with findings in previous studies [11, 23, 24]. By tracking plasmids carrying $b / a_{\mid \mathrm{MP}-6}$, we clarified the relationships between $b / a_{\mathrm{MPP}}$-harbouring isolates that seemed diverse based on Xbal-PFGE analysis or comparison of short-read WGS results.

The current study revealed predominant dissemination of pKPI-6 in the study area, which may have resulted in the emergence of heterogeneous subpopulations. Group IncF plasmids possessed similar genomic structures, consisting of the globally disseminated IncF plasmid and a $b / a_{\mid \mathrm{MP}-6}$ cassette cointegrated on the pKPI-6 genome, without accompaniment of bla $\mathrm{CTX}_{\mathrm{M}-2}$ (Figure S6). Our analysis revealed that $b / a_{\mid \mathrm{MP}-6}$ transcription was lower from Group IncF plasmid (pE305_IMP6 single) than from pKPI-6 in E. coli cells of the same genetic background (Supplementary Figure S8). Low carbapenemase gene transcription is considered as one of the reasons for reduced resistance to meropenem [25]. Therefore, CRE isolates carrying Group IncF plasmids might have a reduced fitness cost for the carriage of $b / a_{1 \mathrm{MP}-6}$, leading to further environmental dissemination of $b / a_{\mathrm{IMP}-6}[26]$.

Unlike for other plasmids in Group IncF, the complete sequence of pE305_IMP6 could not be obtained by long-read or short-read sequencing because of a signature 19-kbp repeat sequence unit. Based on combined WGS, Southern blotting, and qPCR data, we proposed a hypothetical structure of pE305_IMP-6 (Fig. 2C). Our results indicated that, despite its clonal origin, CRE isolate E305 comprised two different populations: a major population carrying pE305_IMP-6 with multiple bla $a_{\mathrm{IMP}-6}$ copies and a minor population carrying pE305_IMP-6 single $_{\text {with }}$ a single bla $a_{\mathrm{MP}-6}$ copy (Supplementary Figure S10). Moreover, the amplification of $b / a_{\mathrm{IMP}-6}$ on the IncF plasmid enhanced the transcription of $b / a_{\mathrm{IMP}-6}$ (Fig. 2E), resulting in increased resistance to meropenem (Supplementary Table S5). These results are consistent with previous studies reporting higher resistance to carbapenem through amplification of $b / a_{0 X A-58}$ [27] and $b / a_{\mathrm{NDM}-1}[19]$.

All E. coli isolates carrying Group IncF plasmids were found to possess $o m p F$ with a premature termination codon (Supplementary Table S3). When an isolate producing wild-type OmpF carries this plasmid with a single copy of $b / a_{\mid \mathrm{MP}-6}$, it is difficult to detect due to weaker resistance to meropenem. However, when an isolate with a porin mutation acquires a Group IncF plasmid with multiple $b / a_{\mid \mathrm{MP}-6}$ copies, it may abruptly exhibit strong resistance to meropenem without any direct trace of horizontal transfer. These types of plasmids may act as "hidden transmitters" of $b / a_{\text {IMP- } 6}$.

Moreover, we demonstrated chromosomal integration of Group IncF plasmids in some E. coli isolates. Carbapenemase genes have been reported to be transmitted primarily through plasmid conjugation [4], and chromosomal integration has been reported in a limited number of strains [28]. In our study, three out of 135 E. coli isolates (2.2\%) exhibited chromosomal integration of bla $a_{\mid \mathrm{MP}-6}$, which presumably occurred during the vast horizontal spread of pKPI-6. Compared with $b / a_{1 \mathrm{MP}-6}$ on plasmids, chromosomal $b / a_{\mathrm{IMP}-6}$ was not readily transmissible to another patient. However, these isolates may stably possess $b / a_{\mid \mathrm{MP}-6}$ within a patient and not lose carbapenem resistance through the elimination of plasmids harbouring $b / a_{\mathrm{IMP}-6}$. 
In the early 1990s, some metallo- $\beta$-lactamases were reported in Japan $[29,30]$, followed by the identification of IMP-1 [31]. Since then, these $\beta$-lactamases have been frequently identified in Japan [32]. The single amino-acid variant, IMP-6, was identified in 2001 [17]. IMP-1 producers have disseminated mainly in eastern Japan, including Tokyo [23, 24,33], whereas IMP-6 producers have been almost exclusively found in western Japan, including Osaka $[7,10,11,24]$. Consistent herewith, in this study, only one $K$. pneumoniae isolate carrying bla $\mathrm{IMP}-1_{1}, \mathrm{E} 105$, was isolated in hospital A, where CRE carrying pKPI-6 were dominant. The patient carrying CRE isolate E105 was hospitalized for 512 days with other inpatients carrying CRE with pKPI-6, and the isolate showed $\sim 83 \%$ similarity with a cluster of $K$. pneumoniae isolates carrying pKPI- 6 in the Xbal-PFGE phylogeny (Fig. 1B). In addition, WGS of the plasmids revealed that a 714-bp region bracketed by 32-bp homologous regions was the only difference between pE105_IMP1 and pE013_IMP6 (Fig. 4A). This very small fragment appeared to have been removed by homologous recombination in pE105_IMP1 (Fig. 4B). Our results suggest that $b / a_{\mathrm{IMP}-6}$ had disseminated via the transmission of pKPI- 6 , and spontaneous mutation may have generated the $b / a_{\mathrm{IMP}-1}$-encoding plasmid providing broader antimicrobial resistance, resulting in increased fitness in the clinical setting.

\section{Conclusions}

This multi-institutional surveillance study uncovered the clonal dissemination of a plasmid encoding a specific carbapenemase IMP- 6 and demonstrated that a seemingly clonal horizontal dissemination of $\mathrm{CRE}$ isolates had embraced heterogeneous minor subpopulations, which exhibited broadened antimicrobial resistance, stable carriage of $b / a_{\mathrm{IMP}-6}$ through chromosomal integration, or heteroresistance related to covert $b / a_{\mathrm{IMP}}$ transmission. Such diverse gene adaptations might also be common among CRE isolates carrying other carbapenemase genes. By focusing on the modes of carbapenemase gene carriage, this study revealed the clonal dissemination of a carbapenemase-encoding plasmid, along with the presence of diverse subpopulations that would ensure and facilitate the dissemination of carbapenemase genes in various environments, resulting in serious complications in clinical settings.

\section{Materials And Methods}

CRE Isolates and PFGE Phylogenetic Analysis. We performed a CRE surveillance study of 1,507 patients hospitalized in 43 hospitals located in northern Osaka between December 2015 and January 2016 [10]. In the current study, we analysed $230 \mathrm{CRE}$ isolates carrying bla $a_{\mathrm{IMP}}$ obtained in the surveillance study, including $135 \mathrm{E}$. coli isolates and $95 \mathrm{~K}$. pneumoniae isolates. All isolates were subjected to Xbal-digested PFGE for phylogenetic analysis [34]. Dendrograms were generated from PFGE patterns by the UPGMA method using BioNumerics software (version 6.6) (Applied Maths NV, Sint-Martens-Latem, Belgium).

Classification of bla ${ }_{\mathrm{IMP}}$ Carriage by PFGE and Southern Blotting. The size and replicon type of $b / a_{1 \mathrm{MP}^{-}}$ harboring plasmids were determined by S1-nuclease-digested PFGE followed by Southern hybridization (S1-nuclease was obtained from Takara Bio, Shiga, Japan). S1-PFGE and Southern blot hybridization for the $b / a_{\mid \mathrm{MP}-6}$ and repA genes encoded on the IncN-type plasmid were performed as described in our 
previous study [35]. The sizes of $b / a_{\mid \mathrm{MP}}$-encoding plasmids were determined using BioNumerics software (version 7.5) (Applied Maths NV). The modes of $b / a_{\mathrm{IMP}}$ carriage were classified into seven groups based on the sizes and replicon types of the plasmids carrying $b / a_{\mathrm{IMP}}$. The groups and their associated characteristics are as follows: Group pKPI-6, a pKPI-6-like bla IMP- 6 -encoding plasmid ( $50 \mathrm{kbp}$, encoding repA for IncN plasmid); Group IncN, a bla $a_{\mathrm{MP}-6}$-encoding plasmid (not $\sim 50 \mathrm{kbp}$, encoding repA for IncN plasmid); Group Non-IncN KP, a b/a $a_{\mathrm{IMP}-6}$-encoding plasmid (without repA for IncN plasmid) harboured by K. pneumoniae isolates; Group IncF, a bla $a_{\mathrm{IMP}-6}$-encoding plasmid (without repA for IncN plasmid) harboured by $E$. coli isolates; Group Double b/a $a_{\mid \mathrm{MP}-6}$, multiple plasmids with $b / a_{\mid \mathrm{MP}-6}$ harboured by a single isolate; Group Chromosome, chromosomal bla $a_{\mathrm{IMP}-6}$; Group Non-Typeable, a b/a $a_{\mathrm{MP}-6}$-encoding plasmid of unknown size; Group IMP1, a bla $\left.\right|_{\mathrm{IMP}-1}$-carrier plasmid.

Isolates classified as chromosomal bla $a_{\mathrm{IMP}}$ carriers were further analysed to identify the location of $b / a_{\mathrm{IMP}}$. In brief, I-Ceul endonuclease-digested PFGE followed by Southern blotting using probes for $b / a_{\mid \mathrm{MP}-6}$ and $16 \mathrm{~S}$ rRNA genes was performed to confirm the location of the bla ${ }_{\mathrm{IMP}}$ gene in three $E$. coli isolates E138, E300, and E302, as previously described [28].

Antimicrobial Susceptibility Testing. Susceptibility to ampicillin, ampicillin/sulbactam, piperacillin/tazobactam, piperacillin, cefotaxime, cefepime, imipenem, and meropenem was determined by the broth microdilution method according to the Clinical and Laboratory Standards Institute document M100-S28 [36]. MICs of meropenem were determined using ETEST ${ }^{\circledR}$ (bioMérieux, Marcy l'Etoile, France), following the manufacturer's instructions.

Whole-Genome Sequencing and Genomic Analysis. Genomic DNA for long- and short-read sequencing was extracted using the DNeasy PowerSoil Kit (Qiagen, Hilden, Germany). Short-read sequencing was conducted on an Illumina HiSeq 3000 sequencer using the KAPA library preparation kit (Kapa Biosystems, Woburn, MA, USA) or on an Illumina MiSeq sequencer using the KAPA HyperPlus Library Preparation Kit (Kapa Biosystems). Long-read sequencing was conducted on a Nanopore GridION sequencer (Oxford Nanopore Technologies, Oxford, UK) using the SQK-LSK109 1D Ligation Sequencing Kit and the EXPNBD103 Native Barcoding Kit. The reads were assembled and polished using Unicycler [37]. In cases where the complete plasmid sequences could not be constructed, sequences were assembled with CANU (version 1.8) [38] or flye [39] and improved using Pilon [40] or Racon [41]. The PlasmidFinder [42] and ResFinder [43] databases were used to identify antimicrobial resistance genes and plasmid replicon types, respectively. A detailed analysis of the insertion sequence was performed using ISfinder [44]. The sequences were annotated with RASTtk [45], and the genomic structures were compared with EasyFig [46]. Plasmids similar to those found in this study were identified using BLAST.

Transformation and Bacterial Transconjugation Assay. Plasmids were prepared from overnight cultures of E. coli isolates $\mathrm{E} 033, \mathrm{E} 066, \mathrm{E} 174$, and $\mathrm{E} 305$, and K. pneumoniae isolates E187, E188, E196, E208, and E328, using the Plasmid Miniprep Kit (Qiagen). Electro-competent TOP10 E. coli cells (Invitrogen, Waltham, MA, USA) were electroporated with the extracted plasmids using a Gene Pulser Xcell System 
(Bio-Rad, Hercules, CA, USA). Following incubation in S.O.C. Medium (Invitrogen) for $2 \mathrm{~h}$ ( $6 \mathrm{~h}$ for isolate E305), transformants were selected on Luria-Bertani (LB) agar supplemented with $0.125 \mu \mathrm{g} / \mathrm{mL}$ meropenem $(2 \mu \mathrm{g} / \mathrm{mL}$ cefotaxime for isolate E305).

Bacterial conjugation assays were performed using the transformants as donors and the sodium azideresistant E. coli strain TUM3456 [47] as a recipient. After mixing overnight cultures of donors and recipients at a $1: 10$ volumetric ratio, the mixture $(10 \mu \mathrm{L})$ was incubated on LB agar for $24 \mathrm{~h}$ at $37^{\circ} \mathrm{C}$. Transconjugants were selected on LB agar containing cefotaxime $(2 \mu \mathrm{g} / \mathrm{mL})$ and sodium azide $(150 \mu \mathrm{g} / \mathrm{mL})$. The conjugation frequency was calculated from the CFU as the number of transconjugants divided by the number of donors plus transconjugants.

Determination of the Plasmid Copy Number per Host Bacterial Cell. DNA of E. coli isolates E305 and E318, and E. coli transformants with plasmids pE188_IMP6 and pE305_IMP6 single (T188 and T305, respectively) was extracted using the DNA Mini Kit (Qiagen). Using qPCR, the copy numbers of the repA2 gene on plasmids pE305_IMP6 and pE318_IMP6 and the b/aIMP-6 gene on pE188_IMP6 were compared with the copy number of the rrsA gene encoding $16 \mathrm{~S}$ ribosomal RNA on the chromosome. qPCRs were carried out using THUNDERBIRD SYBR qPCR Mix (TOYOBO Life Science, Osaka, Japan) on a LightCycler 96 System (Roche Life Science, Penzberg, Germany). Primers used for this assay are list in Table S8. qPCR analysis was performed using data from repeated experiments $(n=6)$, and the plasmid copy number per cell was calculated from Ct values using the comparative $\mathrm{Ct}$ method [48].

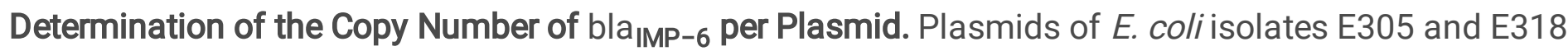
were extracted using the Plasmid Miniprep Kit (Qiagen). Using qPCR, the copy numbers of the $b / a_{\mid M P-6}$ gene were compared with those of the repA2 gene on plasmids pE305_IMP6 and pE318_IMP6. qPCRs were carried out using THUNDERBIRD SYBR qPCR Mix on a LightCycler 96 System. Primers used for this assay are listed in Table S8. qPCR analysis was performed using data from repeated experiments $(n=5)$, and the $b / a_{\mid \mathrm{MP}-6}$ copy number per plasmid was calculated from $\mathrm{Ct}$ values using the comparative $\mathrm{Ct}$ method.

Transcription of bla ${ }_{\mathrm{IMP}-6}$. E. coli isolates E305 and E318, and E. colitransformants T188 and T305 were incubated in LB broth until the optical density at $600 \mathrm{~nm}\left(\mathrm{OD}_{600}\right)$ reached 0.3-0.4. Total RNA was extracted using the RNeasy Mini Kit (Qiagen). RNA was treated with ReverTra Ace qPCR RT Master Mix with gDNA Remover (TOYOBO Life Science) to remove contaminating DNA and to reverse-transcribe the RNA into cDNA. For quality control, DNase-treated RNA that had not been reverse-transcribed was subjected to a DNA contamination test by qPCR. The rrsA gene encoding $16 \mathrm{~S}$ ribosomal RNA served as an endogenous control for normalization. qPCRs were carried out using THUNDERBIRD SYBR qPCR Mix on a LightCycler 96 System. Primers used for this assay are listed in Table S8. qPCR analysis was performed using data from repeated experiments $(n=7)$, and transcript levels were calculated from $\mathrm{Ct}$ values using the comparative $\mathrm{Ct}$ method.

\section{Declarations}


Acknowledgements

The authors thank Isao Nishi in Osaka University hospital for supporting antimicrobial resistance assays, and Yoshikazu Ishii in Toho University Graduate School of Medicine for providing E. coli TUM3456.

\section{Funding}

This work was supported by the Japan Initiative for Global Research Network on Infectious Diseases (JGRID) from Ministry of Education, Culture, Sport, Science and Technology in Japan; and the Japan Agency for Medical Research and Development (AMED) [grant number 19fm0108003h0005]

\section{Availability of data and materials}

The WGS data are available from the DDBJ (DNA Data Bank of Japan) database under the following accession numbers; AB616660, AP19402, AP19405, and AP022349 to AP022369. Raw data of isolate E305 are available at NCBI under accession numbers DRX184368 and DRX182679.

\section{Authors' contributions}

R.A. and Y.A. designed research; R.A. and R.K. performed research; R.A., Y.A., Y.S., D.T., Y.M., and D.M. analysed data; R.A., Y.A., N.Y., K.T., Y.F., and S.H wrote the paper. All authors edited and approved of the final manuscript.

\section{Ethics approval and consent to participate}

Not applicable.

\section{Consent for publication}

Not applicable.

\section{Competing interest}

The authors declare that they have no competing interests.

\section{References}

1. World Health Organization. Antimicrobial resistance: global report on surveillance. 2014. https://apps.who.int/iris/handle/10665/112642. Accessed 21 May 2020.

2. Logan LK, Weinstein RA. The epidemiology of carbapenem-resistant Enterobacteriaceae: the impact and evolution of a global menace. J Infect Dis. 2017;215:28-36.

3. Nordmann P, Naas T, Poirel L. Global spread of carbapenemase-producing Enterobacteriaceae. Emerg Infect Dis. 2011;17:1791-8. 
4. Kopotsa K, Osei Sekyere J, Mbelle NM. Plasmid evolution in carbapenemase-producing Enterobacteriaceae: a review. Ann N Y Acad Sci. 2019;1457:61-91.

5. Kanamori H, Parobek CM, Juliano JJ, van Duin D, Cairns BA, Weber DJ, et al. A prolonged outbreak of KPC-3-producing Enterobacter cloacae and Klebsiella pneumoniae driven by multiple mechanisms of resistance transmission at a large academic burn center. Antimicrob Agents Chemother. 2017;61:e01516-16.

6. $10.1093 / \mathrm{cid} / \mathrm{ciaa} 049 / 5817041$

de Man TJB, Yaffee AQ, Zhu W, et al. Multispecies outbreak of Verona integron-encoded metallo-ßlactamase-producing multidrugresistant bacteria driven by a promiscuous incompatibility group A/C2. Clin Infect Dis. 2020. https://academic.oup.com/cid/advance-articleabstract/doi/10.1093/cid/ciaa049/5817041. Accessed 22 May 2020.

7. $10.1038 / s 41598-020-60659-2$

Yamagishi T, Matsui M, Sekizuka T, Ito H, Fukusumi M, Uehira T, et al. A prolonged multispecies outbreak of IMP-6 carbapenemase-producing enterobacterales due to horizontal transmission of the IncN plasmid. Sci Rep. 2020;10:4149. Available at: https://doi.org/10.1038/s41598-020-60659-2. Accessed 22 May 2020.

8. Mathers AJ, Peirano G, Pitout JDD. The role of epidemic resistance plasmids and international highrisk clones in the spread of multidrug-resistant Enterobacteriaceae. Clin Microbiol Rev. 2015;28:56591.

9. Harris PNA, Alexander MW. Beyond the core tenome: tracking plasmids in outbreaks of multidrugresistant bacteria. Clin Infect Dis. 2020;1:1-2.

10. Yamamoto N, Asada R, Kawahara R, Hagiya H, Akeda Y, Shanmugakani RK, et al. Prevalence of, and risk factors for, carriage of carbapenem-resistant Enterobacteriaceae among hospitalized patients in Japan. J Hosp Infect. 2017;97:212-7.

11. Kayama S, Shigemoto N, Kuwahara R, Oshima K, Hirakawa H, Hisatsune J, et al. Complete nucleotide sequence of the IncN plasmid encoding IMP-6 and CTX-M-2 from emerging carbapenem-resistant Enterobacteriaceae in Japan. Antimicrob Agents Chemother. 2015;59:1356-9.

12. Weingarten RA, Johnson RC, Conlan S, Ramsburg AM, Dekker JP, Lau AF, et al. Genomic analysis of hospital plumbing reveals diverse reservoir of bacterial plasmids conferring carbapenem resistance. MBio. 2018;9:e02011-17.

13. Liu L, Feng Y, Long H, McNally A, Zong Z. Sequence type 273 carbapenem-resistant Klebsiella pneumoniae carrying $b / a_{\mathrm{NDM}-1}$ and b/a $a_{\mathrm{MP}-4}$. Antimicrob Agents Chemother. 2018;62:e00161-18.

14. Yang $L$, Lin $Y, L u L, X u e ~ M, M a ~ H, ~ G u o ~ X$, et al. Coexistence of two bla $a_{N D M-5}$ genes carried on IncX3 and IncFIl plasmids in an Escherichia coli isolate revealed by Illumina and Nanopore sequencing. Front Microbiol. 2020;11:195.

15. Rahube TO, Viana LS, Koraimann G, Yost CK. Characterization and comparative analysis of antibiotic resistance plasmids isolated from a wastewater treatment plant. Front Microbiol. 2014;5:558. 
16. Nikaido $\mathrm{H}$. Molecular basis of bacterial outer membrane permeability revisited. Microbiol Mol Biol Rev. 2003;67:593-656.

17. Yano H, Kuga A, Okamoto R, Kitasato H, Kobayashi T, Inoue M. Plasmid-encoded metallo-betalactamase (IMP-6) conferring resistance to carbapenems, especially meropenem. Antimicrob Agents Chemother. 2001;45:1343-8.

18. Wick RR, Schultz MB, Zobel J, Holt KE. Bandage: interactive visualization of de novo genome assemblies. Bioinformatics. 2015;31:3350-2.

19. Huang T-W, Chen T-L, Chen Y-T, Lauderdale T-L, Liao T-L, Lee $Y-T$, et al. Copy number change of the NDM-1 sequence in a multidrug-resistant Klebsiella pneumoniae clinical isolate. PLoS One. 2013;8:e62774.

20. Rodriguez M, Holcik M, Iyer VN. Lethality and survival of Klebsiella oxytoca evoked by conjugative IncN group plasmids. J Bacteriol. 1995;177:6352-61.

21. Sherry NL, Lane CR, Kwong JC, Schultz M, Sait M, Stevens K, et al. Genomics for molecular epidemiology and detecting transmission of carbapenemase-producing Enterobacterales in Victoria, Australia, 2012 to 2016. J Clin Microbiol. 2019;57.

22. Zhang X, Chen D, Xu G, Huang W, Wang X. Molecular epidemiology and drug resistant mechanism in carbapenem-resistant Klebsiella pneumoniae isolated from pediatric patients in Shanghai, China. PLoS One. 2018;13:e0194000.

23. Hayakawa K, Miyoshi-Akiyama T, Kirikae T, Nagamatsu M, Shimada K, Mezaki K, et al. Molecular and epidemiological characterization of IMP-type metallo-beta-lactamase-producing Enterobacter cloacae in a large tertiary care hospital in Japan. Antimicrob Agents Chemother. 2014;58:3441-50.

24. Ohno Y, Nakamura A, Hashimoto E, Matsutani H, Abe N, Fukuda S, et al. Molecular epidemiology of carbapenemase-producing Enterobacteriaceae in a primary care hospital in Japan, 2010-2013. J Infect Chemother. 2017;23:224-9.

25. Nicoloff H, Hjort K, Levin BR, Andersson DI. The high prevalence of antibiotic heteroresistance in pathogenic bacteria is mainly caused by gene amplification. Nat Microbiol. 2019;4:504-14.

26. Andersson DI, Hughes D. Antibiotic resistance and its cost: is it possible to reverse resistance? Nat Rev Microbiol. 2010;8:260-71.

27. Bertini A, Poirel L, Bernabeu S, Fortini D, Villa L, Nordmann P, et al. Multicopy bla $0 x A-58$ gene as a source of high-level resistance to carbapenems in Acinetobacter baumannii. Antimicrob Agents Chemother. 2007;51:2324-8.

28. Sakamoto N, Akeda Y, Sugawara Y, Takeuchi D, Motooka D, Yamamoto N, et al. Genomic characterization of carbapenemase-producing Klebsiella pneumoniae with chromosomally carried blaNDM-1. Antimicrob Agents Chemother. 2018;62.

29. Watanabe M, lyobe S, Inoue M, Mitsuhashi S. Transferable imipenem resistance in Pseudomonas aeruginosa. Antimicrob Agents Chemother. 1991;35:147-51. 
30. Bandoh K, Watanabe K, Muto Y, Tanaka Y, Kato N, Ueno K. Conjugal transfer of imipenem resistance in Bacteroides fragilis. J Antibiot (Tokyo). 1992;45:542-7.

31. Osano E, Arakawa Y, Wacharotayankun R, Ohta M, Horii T, Ito H, et al. Molecular characterization of an enterobacterial metallo beta-lactamase found in a clinical isolate of Serratia marcescens that shows imipenem resistance. Antimicrob Agents Chemother. 1994;38:71-8.

32. Kurokawa H, Yagi T, Shibata N, Shibayama K, Arakawa Y. Worldwide proliferation of carbapenemresistant gram-negative bacteria. Lancet. 1999;354:955.

33. Aoki K, Harada S, Yahara K, Ishii Y, Motooka D, Nakamura S, et al. Molecular characterization of IMP1-producing Enterobacter cloacae complex isolates in Tokyo. Antimicrob Agents Chemother. 2018;62:e02091-17.

34. Aoki K, Harada S, Yahara K, Ishii Y, Motooka D, Nakamura S, et al. Molecular characterization of IMP1-producing Enterobacter cloacae complex isolates in Tokyo. Antimicrob Agents Chemother. 2018;62:e02091-17.

35. Pei Y, Terajima J, Saito Y, Suzuki R, Takai N, Izumiya H, et al. Molecular characterization of enterohemorrhagic Escherichia coli 0157:H7 isolates dispersed across Japan by pulsed-field gel electrophoresis and multiple-locus variable-number tandem repeat analysis. Jpn J Infect Dis. 2008;61:58-64.

36. Abe R, Akeda Y, Sakamoto N, Kumwenda G, Sugawara Y, Yamamoto N, et al. Genomic characterisation of a novel plasmid carrying $b / a_{\mathrm{IMP}-6}$ of carbapenem-resistant Klebsiella pneumoniae isolated in Osaka, Japan. J Glob Antimicrob Resist. 2019;21:195-9.

37. Clinical and Laboratory Standards Institute. M100 Performance Standards for Antimicrobial Susceptibility Testing, 28th edition. http://file.qums.ac.ir/repository/mmrc/CLSI-2018-M100-S28.pdf. Accessed 21 May 2020.

38. Wick RR, Judd LM, Gorrie CL, Holt KE. Unicycler: Resolving bacterial genome assemblies from short and long sequencing reads. PLoS Comput Biol. 2017;13:e1005595.

39. Koren S, Walenz BP, Berlin K, Miller JR, Bergman NH, Phillippy AM. Canu: scalable and accurate longread assembly via adaptive k-mer weighting and repeat separation. Genome Res. 2017;27:722-36.

40. Kolmogorov M, Yuan J, Lin Y, Pevzner PA. Assembly of long, error-prone reads using repeat graphs. Nat Biotechnol. 2019;37:540-6.

41. Walker BJ, Abeel T, Shea T, Priest M, Abouelliel A, Sakthikumar S, et al. Pilon: an integrated tool for comprehensive microbial variant detection and genome assembly improvement. PLoS One. 2014;9:e112963.

42. Vaser R, Sovic I, Nagarajan N, Sikic M. Fast and accurate de novo genome assembly from long uncorrected reads. Genome Res. 2017;27:737-46.

43. Carattoli A, Zankari E, Garcia-Fernandez A, Voldby Larsen M, Lund O, Villa L, et al. In silico detection and typing of plasmids using PlasmidFinder and plasmid multilocus sequence typing. Antimicrob Agents Chemother. 2014;58:3895-903. 
44. Zankari $\mathrm{E}$, Hasman $\mathrm{H}$, Cosentino $\mathrm{S}$, Vestergaard $\mathrm{M}$, Rasmussen $\mathrm{S}$, Lund $\mathrm{O}$, et al. Identification of acquired antimicrobial resistance genes. J Antimicrob Chemother. 2012;67:2640-4.

45. Siguier P, Perochon J, Lestrade L, Mahillon J, Chandler M. ISfinder: the reference centre for bacterial insertion sequences. Nucleic Acids Res. 2006;34:D32-6.

46. Brettin T, Davis JJ, Disz T, Edwards RA, Gerdes S, Olsen GJ, et al. RASTtk: a modular and extensible implementation of the RAST algorithm for building custom annotation pipelines and annotating batches of genomes. Sci Rep. 2015;5:8365.

47. Sullivan MJ, Petty NK, Beatson SA. Easyfig: a genome comparison visualizer. Bioinformatics. 2011;27:1009-10.

48. Ma L, Ishii Y, Ishiguro M, Matsuzawa H, Yamaguchi K. Cloning and sequencing of the gene encoding Toho-2, a class A beta-lactamase preferentially inhibited by tazobactam. Antimicrob Agents Chemother. 1998;42:1181-6.

49. Schmittgen TD, Livak KJ. Analyzing real-time PCR data by the comparative CT method. Nat Protoc. 2008;3:1101-8.

\section{Figures}



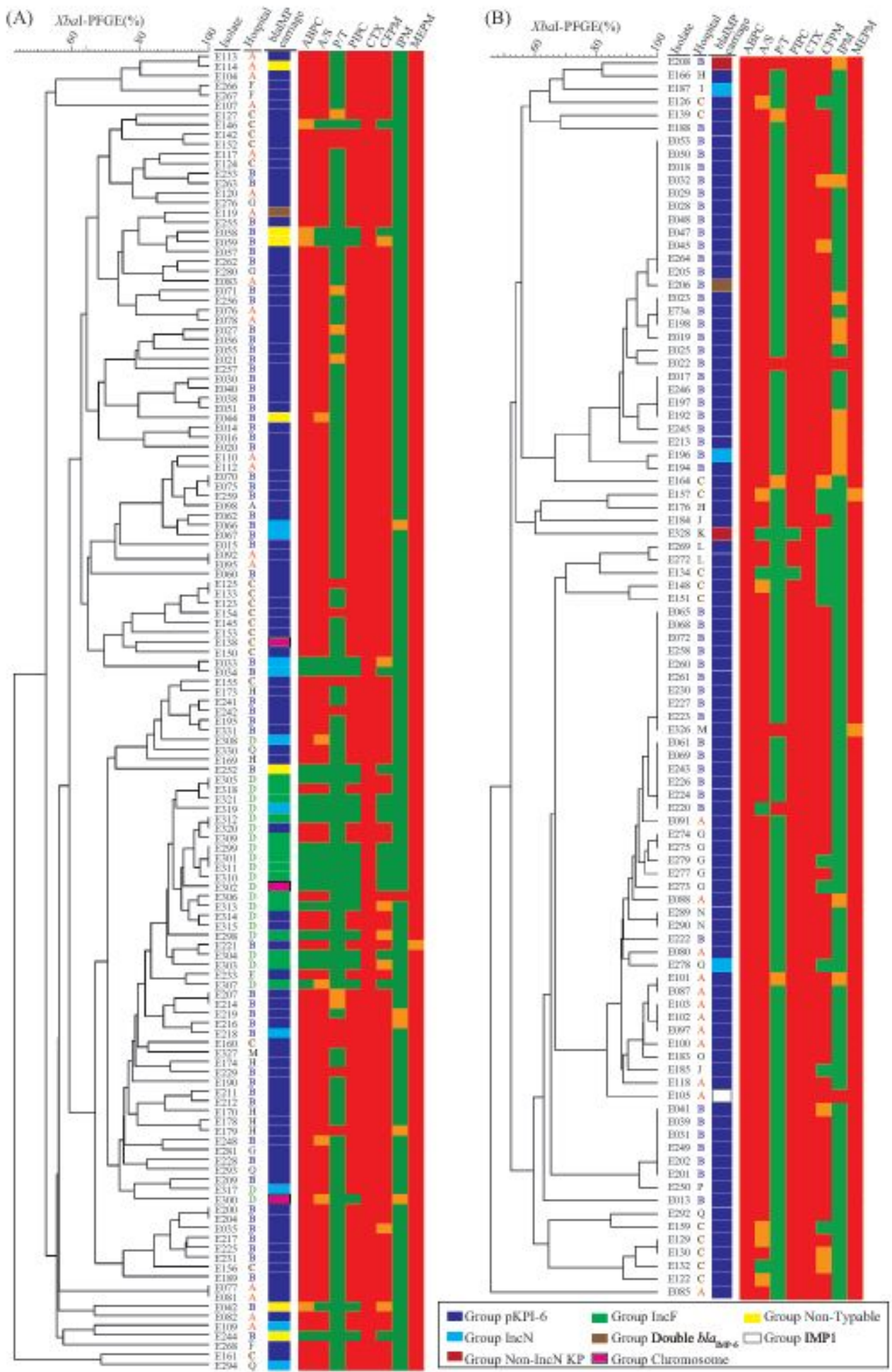

\section{Figure 1}

Phylogenetic trees based on Xbal-PFGE and classification of blalMP carriage and antimicrobial resistance patterns. blalMP carriage of (A) E. coli and (B) K. pneumoniae isolates was classified according to the size and replicon type of the blaIMP-carrier plasmids, determined by S1-PFGE and Southern blotting for blaIMP- 6 and repA on the IncN plasmid. blaIMP carriage was classified designated as follows: blue, Group pKPI-6, pKPI-6-like plasmid ( 50 kbp, encoding repA for IncN plasmid); light blue, 
Group IncN, plasmid with repA for IncN, but not $~ 50$ kbp; red, Group Non-IncN KP, plasmid without repA for IncN harboured by K. pneumoniae; green, Group IncF, plasmid without repA for IncN harboured by E. coli; brown, Group Double blaIMP-6, multiple plasmids with blaIMP-6 carried by a single isolate; enclosed pink, Group Chromosome, chromosomal blaIMP-6; yellow, Group Non-Typeable, failure to determine the size of plasmid carrying blaIMP-6; white, Group IMP1, blaIMP-1-carrier plasmid. Hospitals where the isolates were obtained are indicated as A to Q. Antimicrobial resistance measured by the broth microdilution method is indicated as follows: red, resistant; orange, intermediate; green, susceptible. Abbreviations: $A B P C$, ampicillin; $A / S$, ampicillin/sulbactam; $P / T$, piperacillin/tazobactam; PIPC, piperacillin; CTX, cefotaxime; CFPM, cefepime; IPM, imipenem; MEPM, meropenem
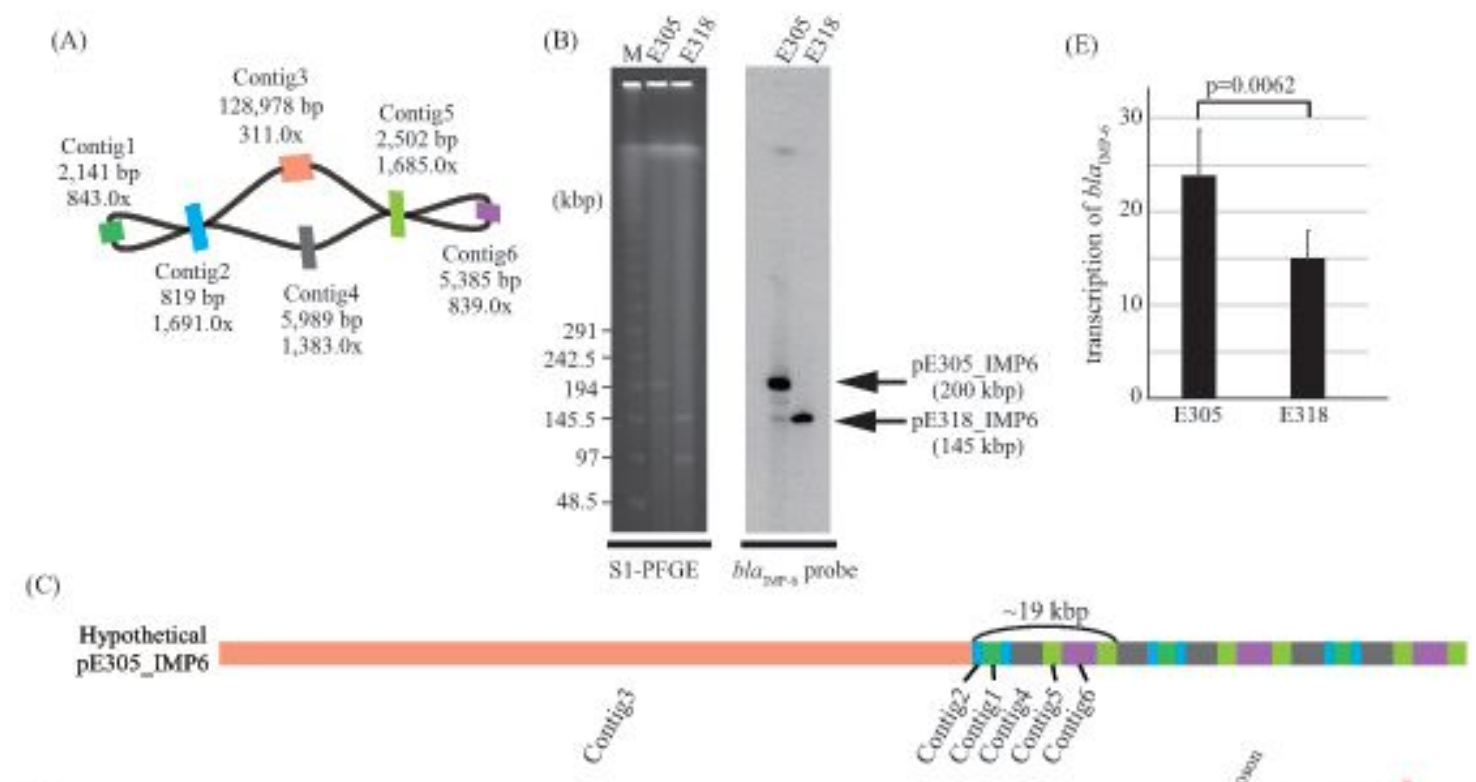

(D)

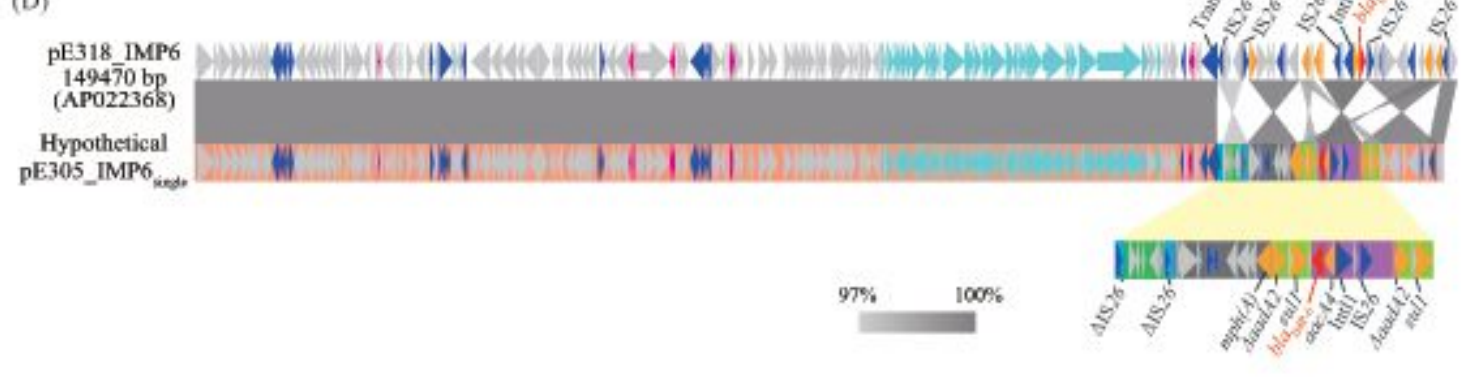

\section{Figure 2}

Genomic structure of Group IncF plasmid pE305_IMP6 and enhanced transcription of blaIMP-6. (A) Genomic structure of plasmid pE305_IMP6. De-novo assembly graph of plasmid pE305_IMP6 visualized by Bandage displays connections between contigs. The length and depth of each contig are shown. Contig2 connects Contig1 with Contig3 or Contig4, and Contig5 connects Contig6 with Contig3 or Contig4. (B) Sizes of plasmids pE305_IMP6 and pE318_IMP6. PFGE of S1-digested genomic DNA from E. coli isolates E305 and E318, followed by Southern blotting with a blaIMP-6 probe indicated the size of each plasmid. M, DNA size marker (lambda ladder; Bio-Rad). (C) Hypothetical structure of pE305_IMP6. 
The colours correspond to the colours of contigs in (A). (D) Genomic comparison of pE318_IMP6 and hypothetical pE305_IMP6single. According to the overlap between contigs of pE305_IMP6, we assembled the hypothetical sequence shown and compared it with the sequence of plasmid pE318_IMP6. Except for the repeating, pE305_IMP6single and pE318_IMP6 were highly similar. Block arrows indicate confirmed or putative open reading frames (ORFs), and their orientations. Arrow size is proportional to the predicted ORF length. The colour code is as follows: red, carbapenem resistance gene; yellow, other antimicrobial resistance gene; light blue, conjugative transfer gene; blue, mobile element; purple, toxin-antitoxin. Putative, hypothetical, or unknown genes are represented as grey arrows. The grey-shaded area indicates regions with high identity between the two sequences. Accession numbers of the plasmids are indicated in brackets. The colours under arrows of pE305_IMP6single correspond to the colours of contigs in (A). (E) Transcript levels of blaIMP-6 in E. coli isolates E305 and E318. qPCR revealed significantly higher transcription of blaIMP-6 in isolate E305 than in isolate E318. The bar chart represents the mRNA transcript ratio of blalMP- 6 to the housekeeping gene rrsA, which was used as a reference gene. Bars indicate the mean \pm standard deviation, calculated from sextuplet experiments. The $p$-value was calculated by the Mann-Whitney U test.
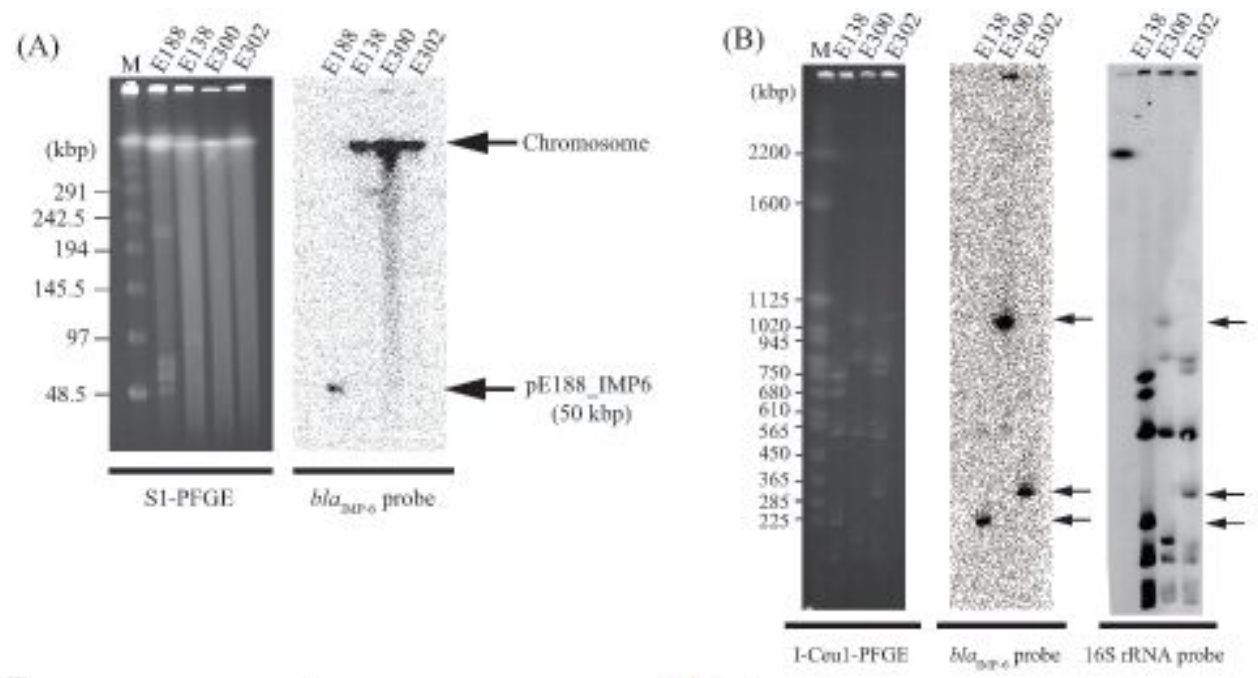

(C)

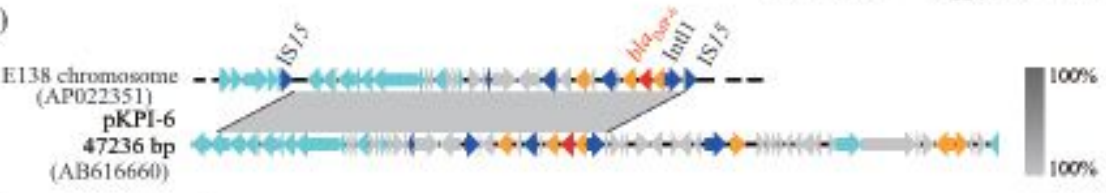

(1)

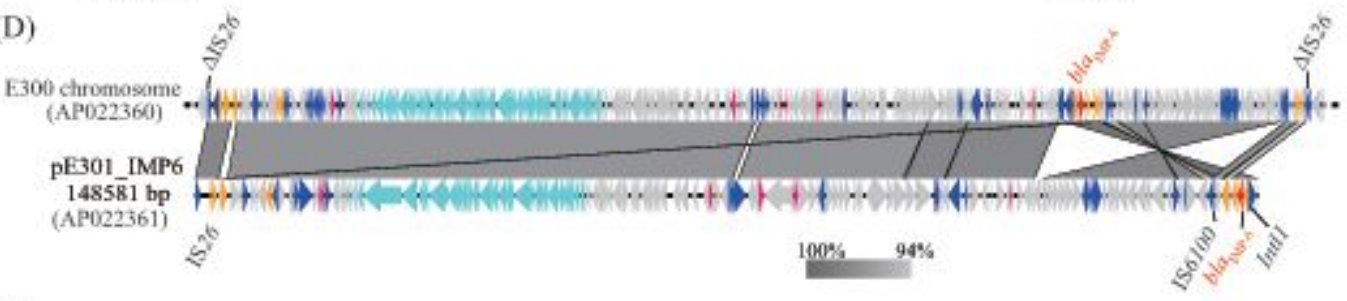

(E)

E302 chromosome
(AP022362)

pE301_IMP6

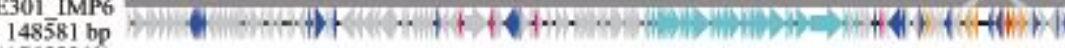

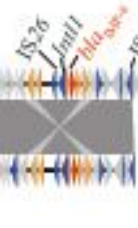

998 (AP022361)

Figure 3 
Chromosomal integration of blaIMP-6. (A) S1-PFGE followed by Southern blotting with a blaIMP-6 probe. Arrows indicate the segments carrying blaIMP-6 in E. coli isolates E138, E300, and E302 (Group Chromosome), and K. pneumoniae isolate E188 (Group pKPI-6). M, DNA size marker (lambda ladder; BioRad). (B) I-Ceu1-PFGE followed by Southern blotting with blaIMP-6 and 16S rRNA probes. Arrows indicate the segments encoding blaIMP-6 or 16S rRNA proving that blaIMP-6 and 16S rRNA were located on the same segment. M, DNA size marker (Saccharomyces cerevisiae ladder; Bio-Rad). (C) Chromosomal integration of a region carrying blalMP-6 in isolate E138. A 23-kbp region containing blalMP-6 of plasmid pKPI-6 was integrated in the chromosome of isolate E138. This region was bracketed by a set of IS15. (D) Comparison of the chromosomal genomic structure of isolate E300 with plasmid pE301_IMP6. Isolate E300 carried chromosomal blaIMP-6, and the region bracketed by a set of mutated IS26 showed high similarity with plasmid pE301_IMP6 in Group IncF. (E) Chromosomal integration of plasmid pE301_IMP6 in isolate E302. Isolate E302 acquired chromosomal blaIMP-6 by incorporation of plasmid pE301_IMP6 bracketed by a set of IS26. The colour code is the same as that described in the legend of Figure 2.

(A)
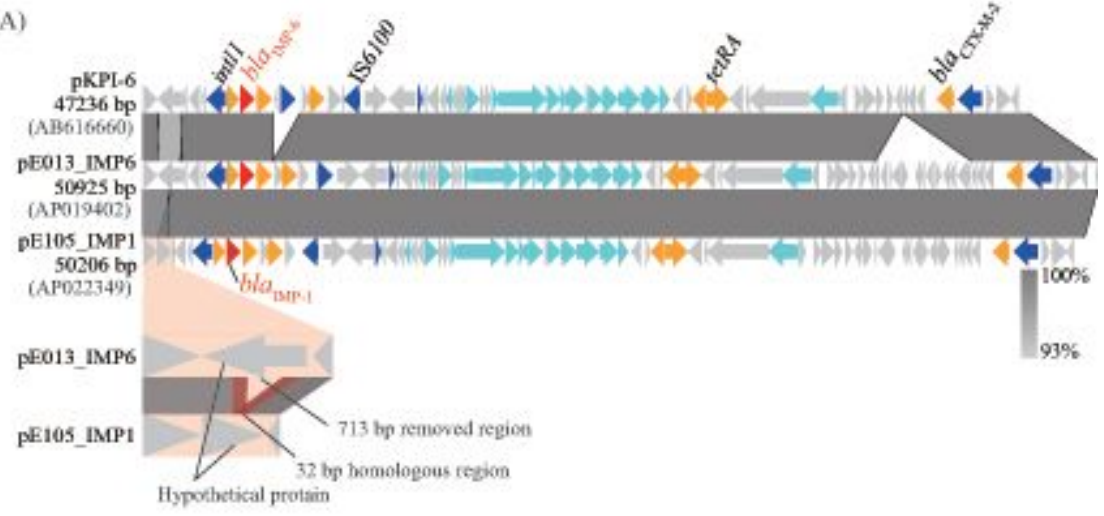

(B)

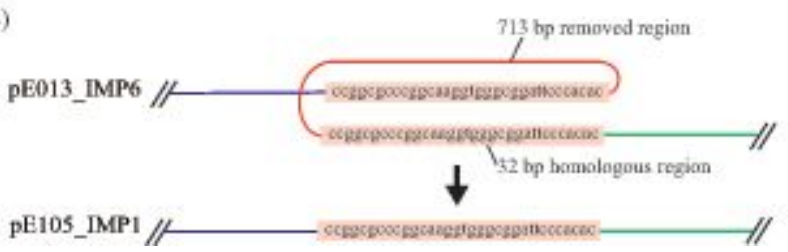

\section{Figure 4}

Plasmid pE105_IMP1 carrying blaIMP-1 was derived from plasmid pKPI-6 by homologous recombination. (A) Comparison of the pE105_IMP1 and pKPI-6 plasmids. The genomic structure of pE105_IMP1 (Group IMP1) was compared with plasmids pKPI-6 and pE013_IMP6 (Group pKPI-6) obtained from K. pneumoniae isolate E013. Differences between pE105_IMP1 and pE013_IMP6 are visually extended at the bottom. The colour code is the same as that described in the legend of Figure 2. (B) Schematic chart of homologous recombination. The 713-bp region of plasmid pE013_IMP6 was removed by homologous recombination at the 32-bp region.

\section{Supplementary Files}

This is a list of supplementary files associated with this preprint. Click to download. 
- Additionalfile18.pdf

- Additionalfile17.pdf

- Additionalfile16.pdf

- Additionalfile15.pdf

- Additionalfile14.pdf

- Additionalfile13.pdf

- Additionalfile12.pdf

- Additionalfile11.pdf

- Additionalfile10.pdf

- Additionalfile9.pdf

- Additionalfile8.pdf

- Additionalfile7.pdf

- Additionalfile6.pdf

- Additionalfile5.pdf

- Additionalfile4.pdf

- Additionalfile3.pdf

- Additionalfile2.pdf

- Additionalfile1.pdf 